\title{
Laparoscopic Distal Gastrectomy in a Patient with Situs Inversus Totalis: A Case Report
}

\author{
Sa-Hong Min ${ }^{1}$, Chang-Min Lee ${ }^{2}$, Heon-Jin Jung ${ }^{1}$, Kyung-Goo Lee ${ }^{1}$, \\ Yun-Suhk Suh ${ }^{1}$, Chung-Il Shin ${ }^{4}$, Hyung-Ho Kim ${ }^{1,2}$, and Han-Kwang Yang ${ }^{1,3}$ \\ ${ }^{1}$ Department of Surgery, Seoul National University College of Medicine, Seoul, \\ ${ }^{2}$ Department of Surgery, Seoul National University Bundang Hospital, Seongnam, \\ ${ }^{3}$ Cancer Research Institute, Seoul National University College of Medicine, \\ ${ }^{4}$ Department of Radiology, Seoul National University Hospital, Seoul, Korea
}

We report our experience with two cases of situs inversus totalis, both involving patients diagnosed with gastric cancer. These were a 52-year-old male with a preoperative staging of CT1bNOMO and a 68-year-old male with a staging of cT2NOMO, both of whom underwent surgery. The former was found to have vascular anomalies in the preoperative computed tomography, so we performed a computed tomography angiography with three-dimensional reconstruction. Laparoscopy-assisted distal gastrectomy with Billroth I anastomosis was performed with D1+ lymph node dissection, and a small laparotomy was made for extracorporeal anastomosis. In contrast, the latter case showed no vascular anomalies in the preoperative computed tomography, and totally laparoscopic distal gastrectomy with delta anastomosis was performed with D1+ lymph node dissection. There were no intraoperative problems in either patient and they were discharged without postoperative complications. Histopathological examination revealed a poorly differentiated adenocarcinoma (pT2NOMO) and a well-differentiated adenocarcinoma (PT1aNOMO), respectively.

Key Words: Situs inversus totalis; Laparoscopic-assisted gastrectomy; Gastric cancer; Lymph node dissection

\section{Introduction}

Situs inversus totalis (SIT) is a rare condition in which there is complete transposition (right to left reversal) of the thoracic and abdominal organs. It is a congenital anomaly that is commonly accompanied by vascular anomalies. ${ }^{1}$ Surgical procedures are technically more complicated in SIT patients due to the different anatomical position of the organs. In 2003, the first case of laparoscopic surgery in for a SIT patient with gastric cancer was reported. ${ }^{2}$ Only

Correspondence to: Han-Kwang Yang

Department of Surgery and Cancer Research Institute, Seoul National University College of Medicine, 101 Daehak-ro, Jongno-gu, Seoul 110744 , Korea

Tel: +82-2-2072-3797, Fax: +82-2-3672-0042

E-mail: hkyang@snu.ac.kr

Received October 4, 2013

Revised October 28, 2013

Accepted October 28, 2013 a few cases of laparoscopy-assisted distal gastrectomy (LADG) for gastric cancer with underlying SIT have been reported in Korea. Moreover, there have been no reports of a total laparoscopic distal gastrectomy (TLDG) with delta anastomosis in a SIT patient. Herein we describe our experience with two patients with SIT who were diagnosed preoperatively with early gastric cancer and who underwent LADG and TLDG with D1+ lymph node dissection. In particular, we outline the preoperative preparation and discuss how the difficulties encountered during the surgery were managed.

\section{Case Report}

\section{Case 1}

In February 2012, a 52-year-old male was diagnosed with early gastric cancer through a screening esophagogastroduodenoscopy (EGD). The patient visited our hospital for further evaluation and 

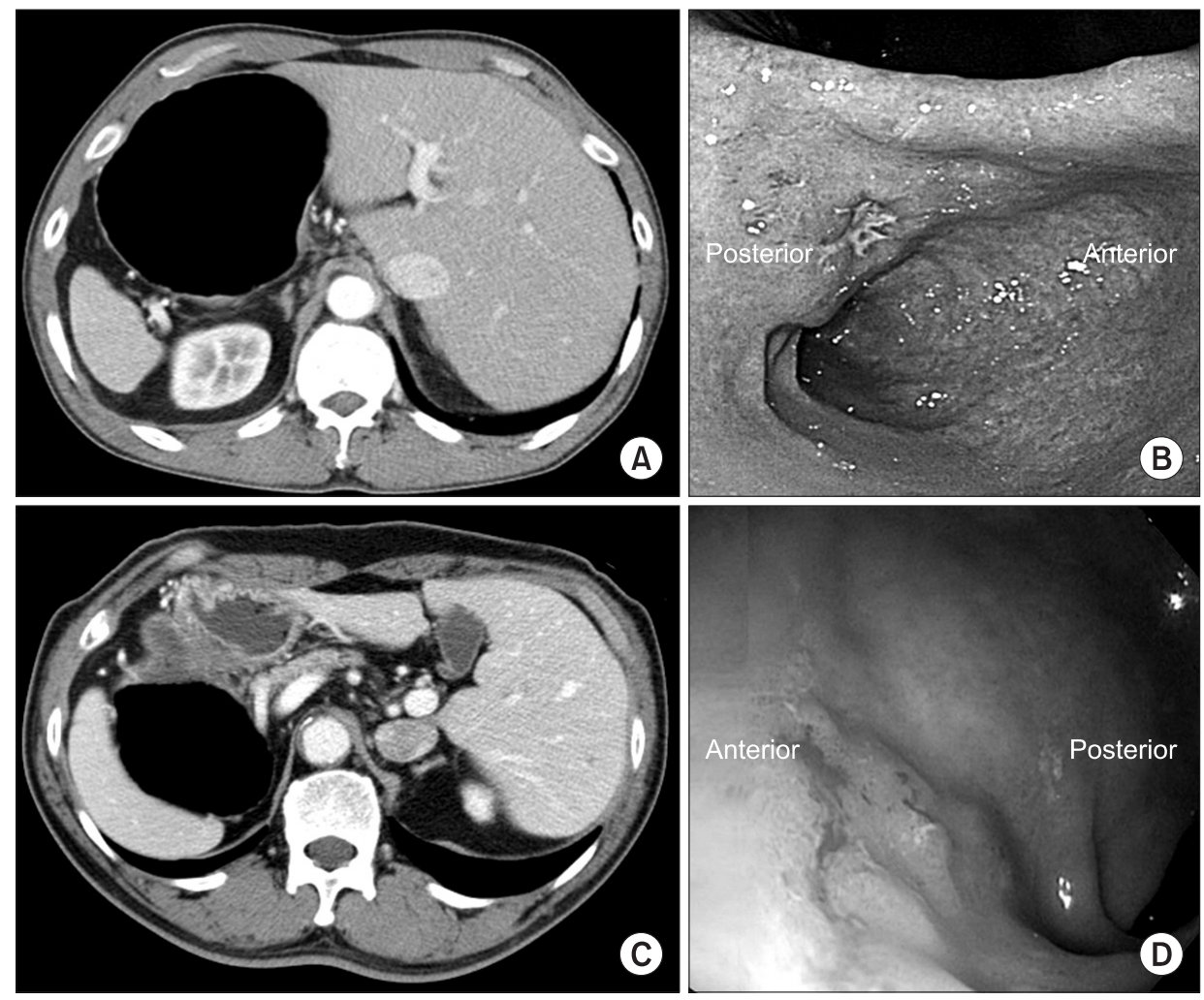

Fig. 1. Computed tomography showing transposition of the abdominal organs in case $1(\mathrm{~A})$, and case $2(\mathrm{C}) .(\mathrm{B}, \mathrm{D})$ Esophagogastroduodenoscopy image showing the lesion on the mid antrum, lesser curvature in case $1(\mathrm{~B})$, and on the mid antrum, anterior wall in case 2 (D).
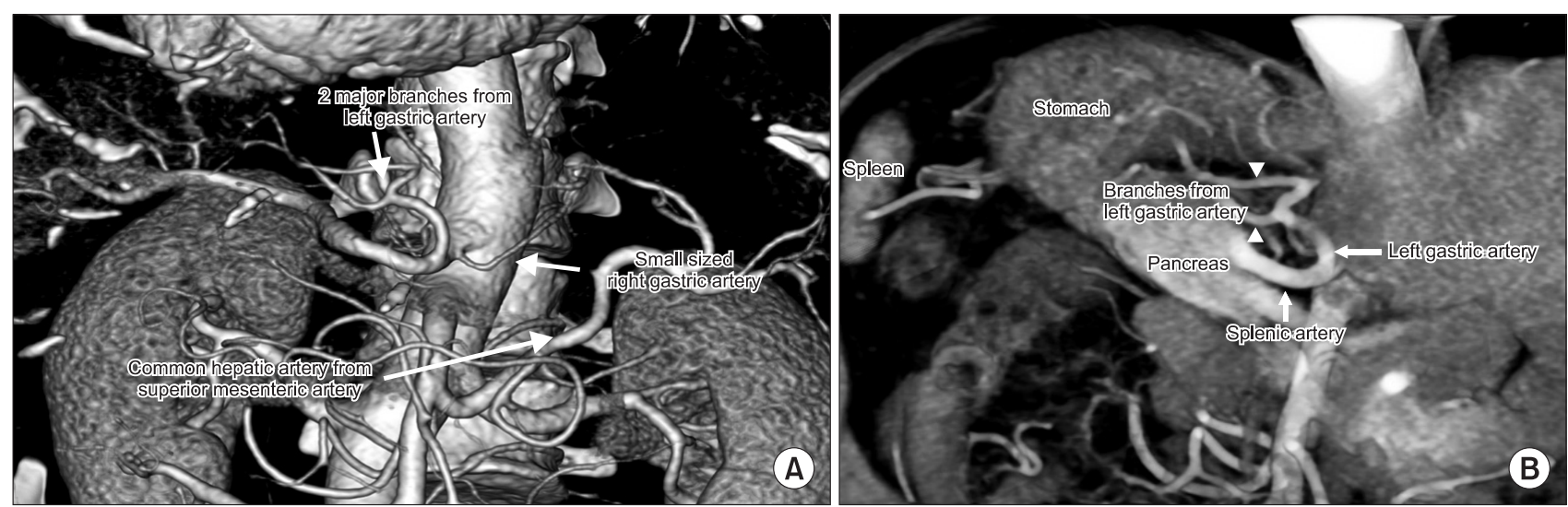

Fig. 2. Case 1. Three-dimensional reconstruction image of computed tomography angiography showing two branches of the left gastric artery (arrowheads), right gastric artery from the celiac trunk, and replaced common hepatic artery from the superior mesenteric artery (A, B).

surgical treatment, if needed. He had been diagnosed with SIT about 30 years previously, but he had not developed any specific disease until this point. His body mass index (BMI) was $22.8 \mathrm{~kg} / \mathrm{m}^{2}$, and he had no other underlying disease and no family history of SIT or stomach cancer. The patient had no history of surgery, except for a right inguinal hernia repair in 2007. Initial vital signs were stable, and a systemic review and physical examination revealed nothing of note. Laboratory examination, including tumor markers, likewise showed no abnormal findings. The chest $\mathrm{x}$-ray film suggested dextrocardia, and an EGD image of the patient indicated an opposite orientation of the pylorus and the stomach (Fig. 1). The lesion was $2 \mathrm{~cm}$ proximal to the pylorus. Preoperative clipping was performed $1 \mathrm{~cm}$ proximal (angle, superjacent, low body anterior wall) and $1 \mathrm{~cm}$ distal (prepyloric antrum) to the ill-defined early gastric cancer lesion on the mid-antrum lesser curvature. Poorly differentiated adenocarcinoma was found on biopsy. Endoscopicultrasonography (EUS) showed submucosal invasion but no definite perigastric lymphadenopathy. Computed tomography (CT) showed transposition of the organs (Fig. 1). There was also focal wall thickening and enhancement in the lesser curvature of the prepy- 


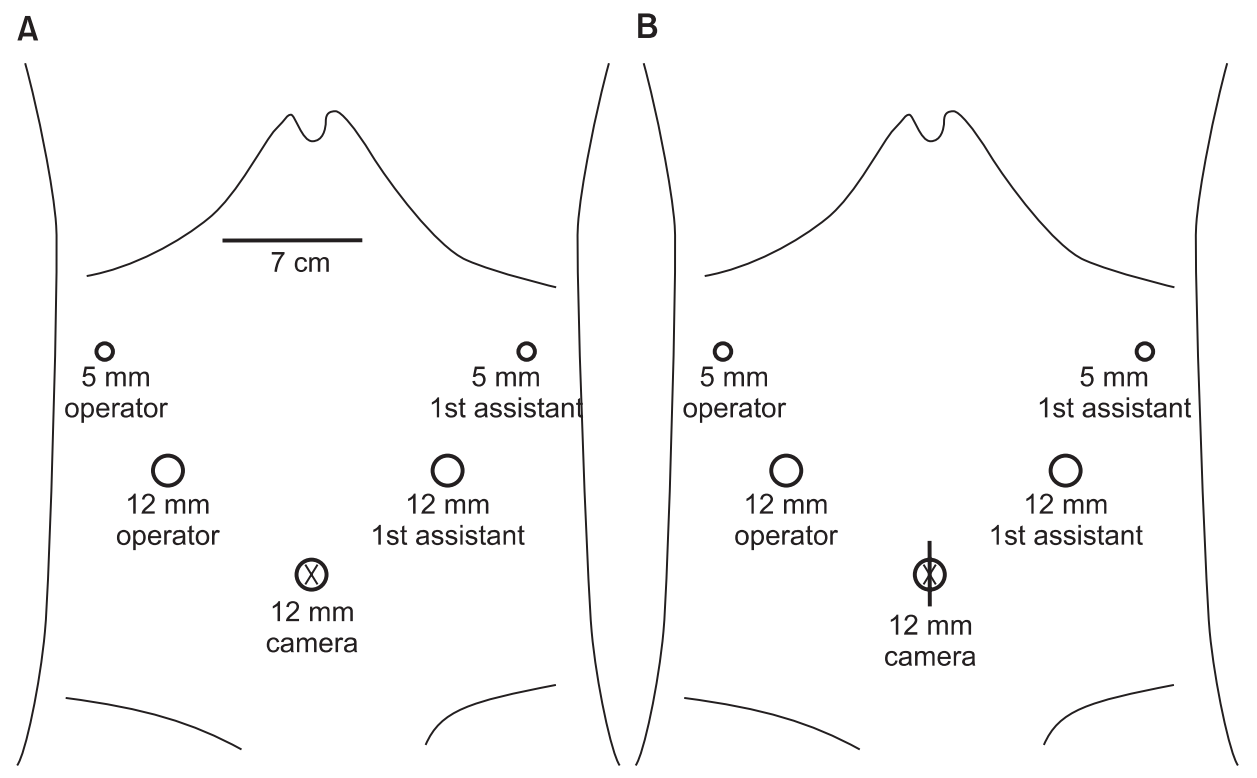

Fig. 3. The placement of ports in (A) case 1 , (B) case 2 . A $7 \mathrm{~cm}$ incision is made below the xyphoid process for extracorporeal anastomosis (A). An extension of the umbilical incision is made for specimen extraction only (B). loric antrum with multiple, tiny lymph nodes in the perilesional and perigastric space.

As there were no distant metastases or visible invasion into adjacent organs, the clinical stage was cT2NOM0, and we decided to perform LADG with D1+ lymph node dissection. Prior to surgery, abdominal angiographic CT with three-dimensional (3D) reconstruction was performed to uncover any variations and to verify the exact structures and locations of the vessels. There were two major artery variations: a replacing common hepatic artery from the superior mesentery artery, and 2 branches from the left gastric artery (Fig. 2).

Laparoscopic surgery was performed. We decided to use the same placement of ports as we do for normal patients (Fig. 3). However, we used a $12 \mathrm{~mm}$ trocar instead of a $5 \mathrm{~mm}$ trocar for the 1st assistant, in case the position of the operator changed. Intraoperatively, there was an obvious transposition of the abdominal organs (Fig. 4). Partial omentectomy was done followed by ligation of the left gastroepiploic vessels. While dissecting the area around the right gastroepiploic vessels and the suprapancreatic portion, the surgeon tried to move to the opposite side, but found that it was easier to perform this dissection on the usual side. The epigastric incision was primarily used as a port for liver retraction before extracorporeal anastomosis. With the liver retracted by the 1st assistant, the lesser omentum was opened. This patient had a common hepatic artery originating from the superior mesenteric artery, so the right gastric artery came from the celiac trunk. Due to its small size, careful dissection and ligation were performed (Fig. 4). Lymph node dissection was performed around the celiac axis and along the lesser curvature. The two branches of the left gastric artery were identified and were found to match the preoperative 3D reconstruction image. The radiologist confirmed the anatomic variation in the surgical field and both branches were safely ligated at the root (Fig. 4). After the D1+ lymph node dissection, an extension of the epigastric port site was made for extracorporeal anastomosis. The now freely movable stomach was brought up to the incision. A modified extracorporeal, double-stapling, end-to-end Billroth I anastomosis procedure was performed (Fig. 4). ${ }^{3}$ The operation time was 220 minutes and there were no intraoperative events, with an estimated blood loss of $100 \mathrm{ml}$. There were no immediate postoperative complications, and the patient was discharged eight days after the operation without any complications.

The final pathology showed a $3.3 \times 2.2 \times 0.3 \mathrm{~cm}$ sized, poorly differentiated Borrmann type 3 lesion with invasion limited to the muscularis propria. There was no metastasis in any of the $29 \mathrm{re}^{-}$ trieved lymph nodes. The final stage was pT2N0, Stage 1B according to the American Joint Committee on Cancer 7th edition.

\section{Case 2}

A 68-year-old male underwent a screening EGD in September of 2012. There were no remarkable findings in the routine laboratory examination including tumor markers, except for an elevated creatinine level $(1.95 \mathrm{mg} / \mathrm{dl})$. This was due to the chronic kidney disease and hypothyroidism for which he was being treated. Despite these conditions, preoperative risk assessment indicated tolerability of a laparoscopic operation. The patient had no history of abdominal surgery. His preoperative BMI was $23.55 \mathrm{~kg} / \mathrm{m}^{2}$, and a 

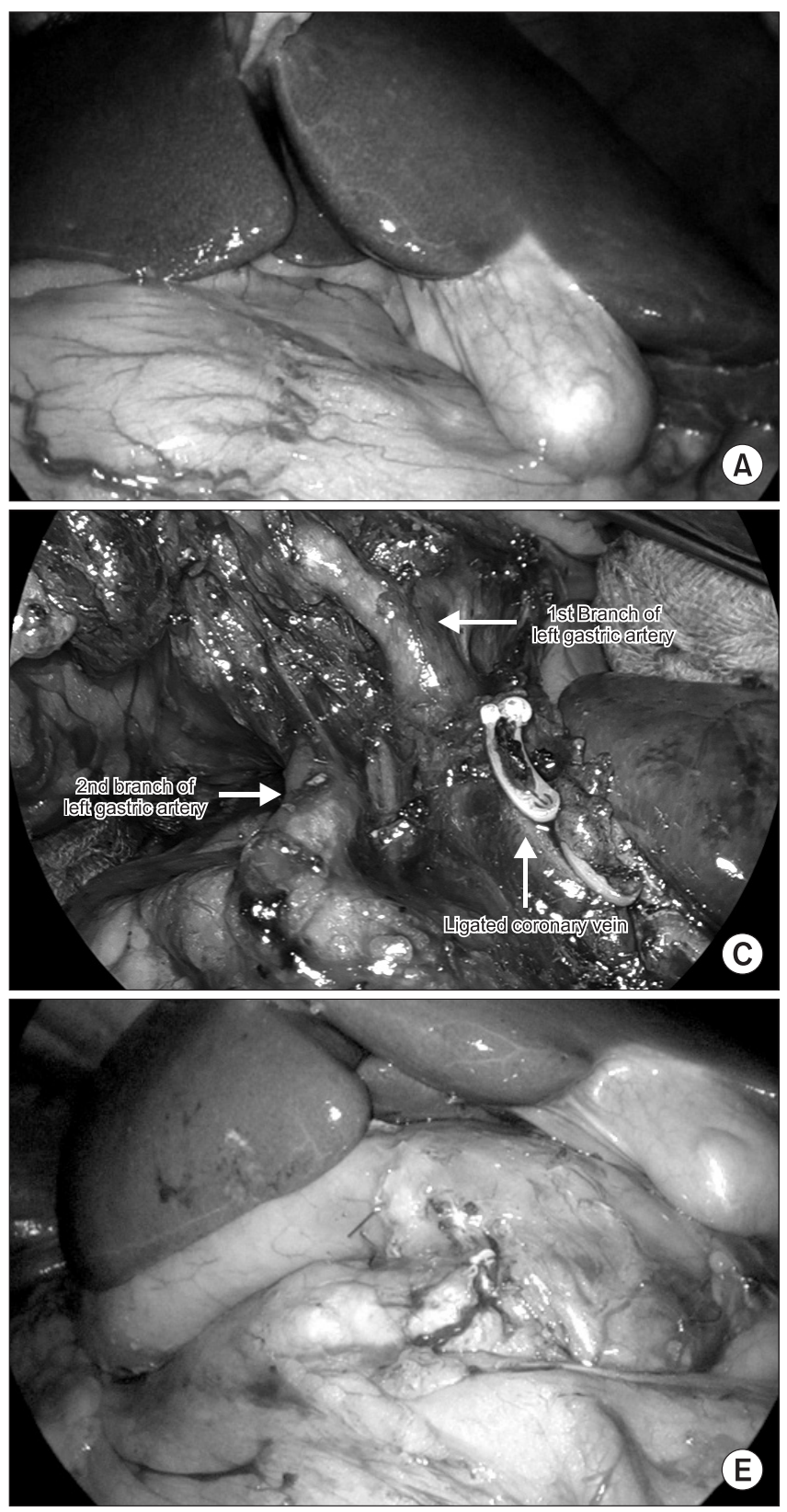

chest $\mathrm{x}$-ray film revealed dextrocardia. EGD showed a hyperemic nodular lesion on the mid antrum anterior wall. Biopsy confirmed well-differentiated adenocarcinoma (Fig. 1). EUS showed definite involvement of the submucosa, but proper muscle layer invasion was suspected. CT showed SIT with no other remarkable findings (Fig. 1). There were no vascular anomalies, and no metastasis or invasion into adjacent structures, so the clinical stage was cT2NOM0. TLDG with delta anastomosis and D1+ lymph node dissection was scheduled. The operator stood on the same side as if there was no SIT throughout the operation. A flexible scope was used through the $12 \mathrm{~mm}$ trocar made on the umbilicus. Two 12 $\mathrm{mm}$ trocars and two $5 \mathrm{~mm}$ trocars were used, making a $\mathrm{V}$ shape
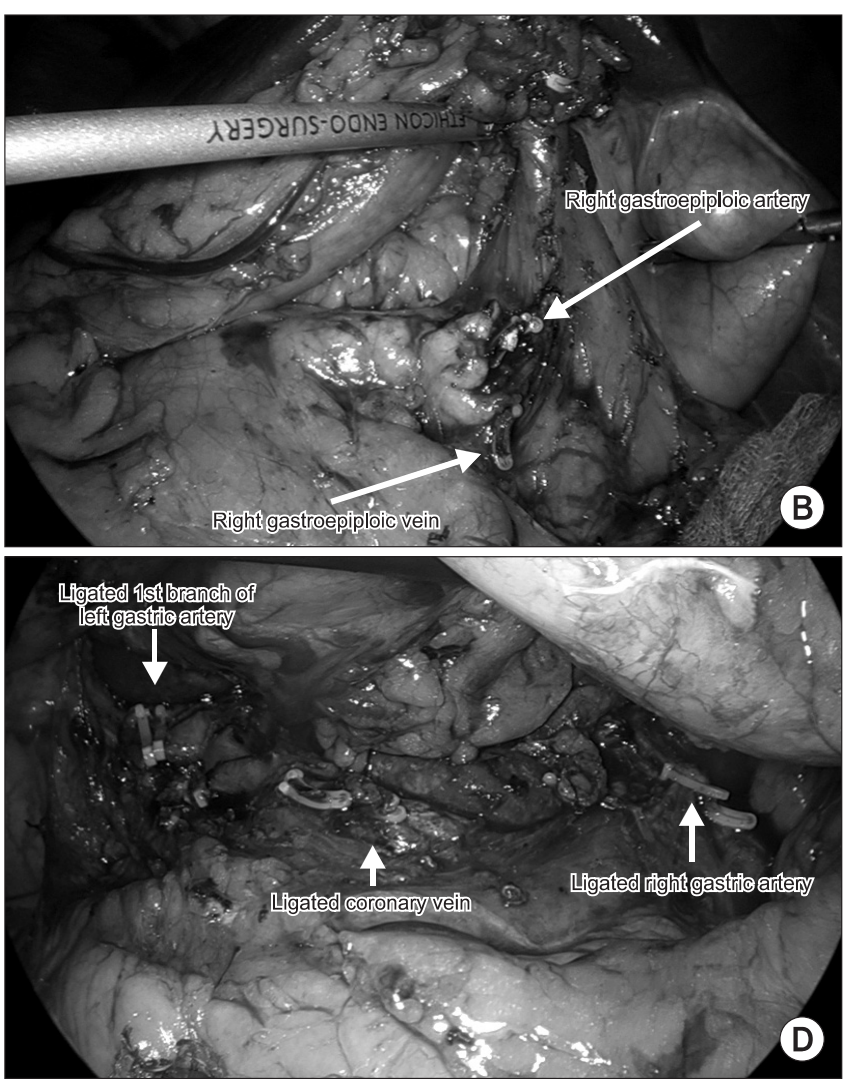

Fig. 4. Case 1. (A) Initial laparoscopic view showing transposition of abdominal organs. (B) The ligated right gastroepiploic artery and vein. The ligated coronary vein. (C) Anatomic variation in the 1st and 2nd branch of the left gastric artery is apparent. (D) The ligated 1st branch of the left gastric artery, right gastric artery and coronary vein. (E) The wound after Billroth I anastomosis and Fibrin glue had been applied.

(Fig. 3). Exposure of the hepatoduodenal ligament and the lesser omentum was achieved with the retraction of the falciform and the left lobe of the liver using a single suture technique. ${ }^{4}$

Partial omentectomy was performed about $4 \mathrm{~cm}$ from the gastroepiploic arcade. The left gastroepiploic vessels were ligated near the fundus and omentectomy was performed along the greater curvature. The right gastroepiploic vessels were then ligated, and the right gastric artery was ligated onto the root from the common hepatic artery. A linear stapler was inserted through the 12 $\mathrm{mm}$ trocar on the right side of the patient, and the duodenum was divided just distal from the pylorus. The left gastric artery and the coronary vein were ligated during lymph node dissection along 

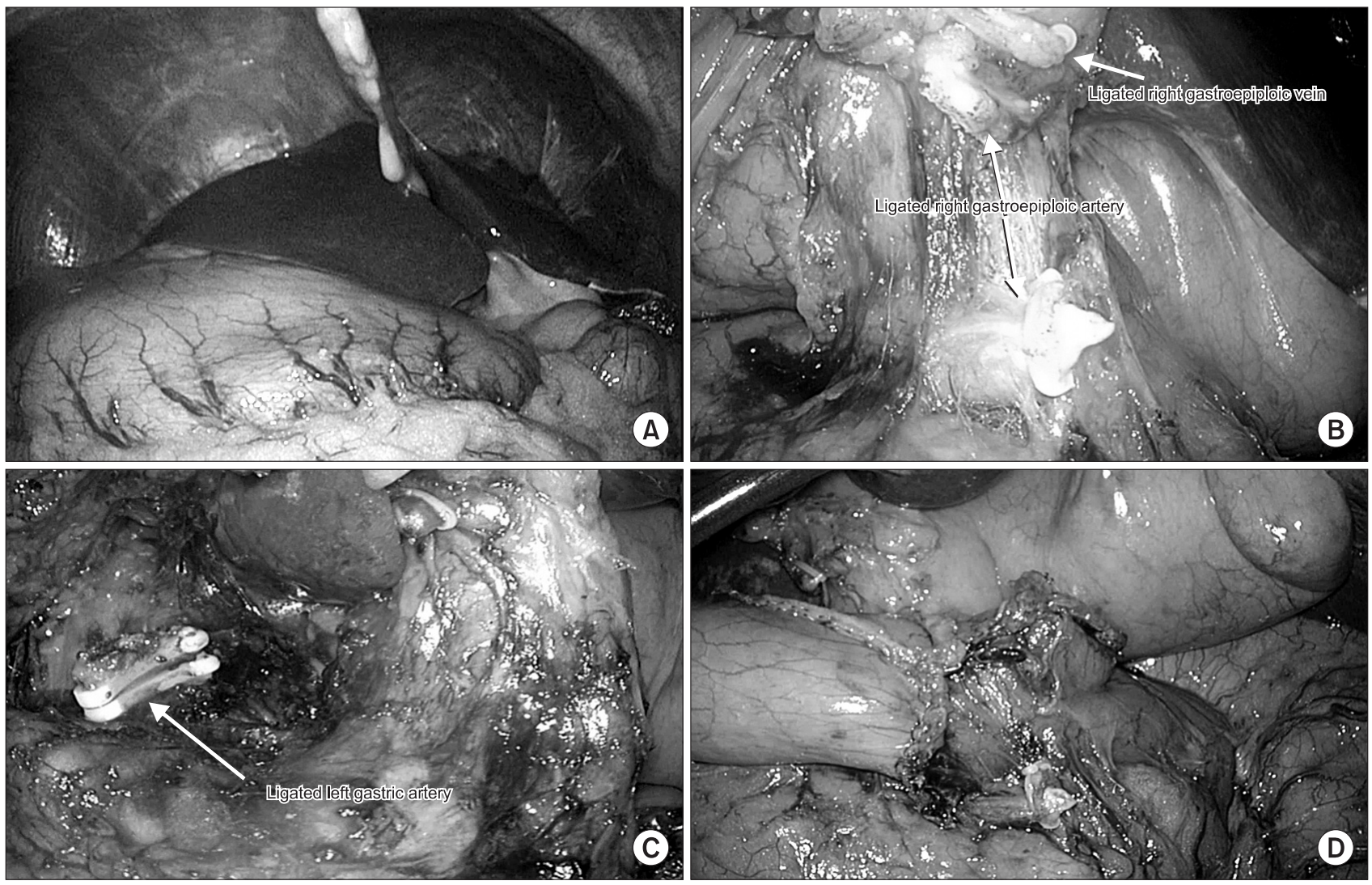

Fig. 5. Case 2. (A) Initial laparoscopic view showing transposition of abdominal organs. (B) Ligated right gastroepiploic artery and vein. (C) Suprapancreatic lymph node dissection with ligated left gastric artery. (D) After delta-shaped anastomosis.

the superior border of the pancreas. After completing lymph node dissection along the lesser curvature, D1+ lymph node dissection was completed. The stomach was divided at the angle portion by a linear stapler and delta-shaped anastomosis was performed (Fig. 5). ${ }^{5}$ The resected specimen was delivered through the umbilicus, with extension of the incision. The operation time was 117 minutes and the estimated blood loss was $50 \mathrm{ml}$, without any intraoperative complications. There were no postoperative complications and the patient was discharged 5 days after the operation.

The final pathology reported a $3.2 \times 1.2 \times 0.1 \mathrm{~cm}$ sized, welldifferentiated adenocarcinoma, localized within the lamina propria. Fifty-nine lymph nodes were retrieved, none of which contained a metastasis. The final pathology was pTlaNO, stage IA.

\section{Discussion}

SIT has been estimated to occur once in every 6,000 to 8,000 births, and forms part of a rare congenital disorder called Kartagener syndrome, along with chronic sinusitis and bronchiectasis. In patients with SIT, all of the chest and abdominal organs are reversed and appear in mirror image when examined or visualized on, for example, an $\mathrm{x}$-ray film.

There is currently no good evidence for SIT being related to gastric cancer. ${ }^{6}$ However, rare synchronous and metachronous multiple primary gastrointestinal malignancies have been reported in the literature. ${ }^{1}$ In 1936, Allen ${ }^{7}$ described the first case of gastrectomy in a gastric cancer patient with SIT. In 2010, LADG with $\mathrm{D} 1+\beta$ lymph-node dissection for early gastric cancer was successfully performed in Japan. It was the first case of laparoscopic gastrectomy with extensive lymph node dissection, and throughout the operation, the monitor was moved to back and forth between the right and left sides while the surgeon stood on the left side, which is opposite to the usual position. In our cases, we performed a D1+ lymph node dissection for early gastric cancer, according to the Japanese classification of gastric cancer. ${ }^{9}$ The surgeon stood on the right side of the patient as usual. Some surgeons might not encounter much difficulty operating in a mirror image position. However, even slight confusion of the anatomy can jeopardize the patient's life, and those who are less experienced with laparoscopic gastrectomy should not take this risk. In these cases, the 1st assis- 
tant is expected to do more, as some of the structures that are easily approached in normal patients are more conveniently accessed by the 1st assistant in patients with SIT. The 2nd assistant, who is mainly in charge of the scope, faces no additional challenge, as the view angle is unchanged. A team discussion before the surgery and excellent coordination between the operator and the 1st assistant is imperative.

There are several reports of laparoscopic gastrectomies in patients with SIT, 2,8,10,11 all of which describe technical difficulties resulting from the unusual anatomy, especially with respect to the vessels. Kang et al. ${ }^{10}$ reported a case of laparoscopy-assisted partial gastrectomy in a patient with SIT and complex vascular anomaly. The right gastric artery originated from the aorta and the common hepatic artery originated from the celiac artery. Reconstruction of vessels using the existing abdominal CT was done for accurate evaluation of the vessels. In our first case, the arterial anomaly was more complex than this (Fig. 2), and using CT alone might have been insufficient to guide the precise ligation of the vessels. We used 3D reconstruction of an abdominal CT angiography image to maximize precision. With this procedure, a smoother operation without major bleeding could be performed. In the second case, no 3D reconstruction of an abdominal CT angiography was performed because there was no vascular anomaly, and the surgery was performed without difficulty. A careful and thorough review of the CT must be performed as a team to prevent surgery-related complications such as bleeding and pancreatic fistulas.

Previous laparoscopy-assisted gastrectomies were performed with a 4 to $7 \mathrm{~cm}$ laparotomy above the anastomosis site. ${ }^{2,8,10,11}$ Likewise the 1st case described here was also a laparoscopy-assisted gastrectomy. A $7 \mathrm{~cm}$ long transverse incision was made below the xyphoid process and above the gastroduodenostomy site. The vascular anomaly did not affect the method of anastomosis. There was no serious difficulty in performing the anastomosis, despite the patient's SIT condition. However, the 2nd case was performed entirely by laparoscopy. Intracorporeal gastroduodenostomy was made with linear staplers only. This was the very first case of intracorporeal delta shaped anastomosis performed in a patient with SIT. No other surgeons have tried a totally laparoscopic subtotal gastrectomy in a patient with SIT before.

In future cases, robotic surgery might be helpful. A single case report on robotic-assisted gastrectomy demonstrated its usefulness. The surgeon does not have to change his position during surgery due to the centered robotic view of the field and it is easy to change instruments in both hands. ${ }^{11}$
In operations for patients with SIT, the most important factor is the anatomy, not only in gastric surgery but also in cholecystectomy and colectomy. If the surgery is performed laparoscopically, the surgical team has to pay extra attention to any anatomic variations. In the case we describe here with vascular anomalies, 3D reconstruction of the CT angiography was very helpful, and indeed we believe that this should be a mandatory procedure in SIT patients with vascular anomalies. It is also important that the operator performs the surgery in the setting with which he most comfortable and best acquainted, in order to reduce unnecessary complications.

\section{Acknowledgments}

This study was supported by $R \& D$ project, the Ministry of Health and Welfare, and the Republic of Korea (Grant No. 80020130183).

\section{References}

1. Iwamura T, Shibata N, Haraguchi Y, Hisashi Y, Nishikawa T, Yamada $\mathrm{H}$, et al. Synchronous double cancer of the stomach and rectum with situs inversus totalis and polysplenia syndrome. J Clin Gastroenterol 2001;33:148-153.

2. Yamaguchi S, Orita H, Yamaoka T, Mii S, Sakata H, Hashizume M. Laparoscope-assisted distal gastrectomy for early gastric cancer in a 76-year-old man with situs inversus totalis. Surg Endosc 2003;17:352-353.

3. Yang HK, Lee HJ, Ahn HS, Yoo MW, Lee IK, Lee KU. Safety of modified double-stapling end-to-end gastroduodenostomy in distal subtotal gastrectomy. J Surg Oncol 2007;96:624-629.

4. Shabbir A, Lee JH, Lee MS, Park do J, Kim HH. Combined suture retraction of the falciform ligament and the left lobe of the liver during laparoscopic total gastrectomy. Surg Endosc 2010;24:3237-3240.

5. Kanaya S, Kawamura Y, Kawada H, Iwasaki H, Gomi T, Satoh $\mathrm{S}$, et al. The delta-shaped anastomosis in laparoscopic distal gastrectomy: analysis of the initial 100 consecutive procedures of intracorporeal gastroduodenostomy. Gastric Cancer 2011;14:365-371.

6. Yoshida Y, Saku M, Masuda Y, Maekawa S, Ikejiri K, Furuyama M. Total gastrectomy for gastric cancer associated with situs inversus totalis. A report of 2 cases. S Afr J Surg 1992;30:156158.

7. Allen FRWK. A case of malignant tumor of the stomach in 
Min SH, et al.

a male with transposition of the viscera. Indian Med Gaz 1936;71:32.

8. Futawatari N, Kikuchi S, Moriya H, Katada N, Sakuramoto S, Watanabe M. Laparoscopy-assisted distal gastrectomy for early gastric cancer with complete situs inversus: report of a case. Surg Today 2010;40:64-67.

9. Sano T, Aiko T. New Japanese classifications and treatment guidelines for gastric cancer: revision concepts and major re- vised points. Gastric Cancer 2011;14:97-100.

10. Kang BH, Lee SL, Hur H, Kim JY, Cho YK, Han SU. Laparoscopy assisted subtotal gastrectomy in gastric cancer patient with situs inversus in Korea. J Korean Surg Soc 2010;79:513517.

11. Kim HB, Lee JH, Park do J, Lee HJ, Kim HH, Yang HK. Robotassisted distal gastrectomy for gastric cancer in a situs inversus totalis patient. J Korean Surg Soc 2012;82:321-324. 